\title{
A Case Study of the Influence of Assessment Attitudes on MOOC Learners' Behavioral Engagement
}

\author{
Kuang Shanyun ${ }^{1, a}$, Xu Zhijun ${ }^{1, b^{*}}$ \\ ${ }^{1}$ Department of Educational Technology, South China Normal University, Guangzhou, China \\ a261869310@qq.com \\ b*469249541@qq.com
}

\begin{abstract}
Students' online behavioral engagement was an important factor that affected their learning performance, and it was also a prerequisite for deep learning. Studies had confirmed that peer assessment activities provided opportunities for students to participate in the formative assessment process, enhanced the interaction between learners and promoted effective learning. However, online learning was more dependent on the learners themselves, and the attitudes of peer assessment might have a greater impact on the assessment process and learning effect. Based on the "Mobile Learning Theory, Technology and Practice", this research used questionnaire surveys and interviews to explore the impact of MOOC learners' assessment attitudes on online behavioral engagement. The results found that: (1) MOOC learners recognized peer assessment, and held a positive attitude. They were willing to take the initiative to participate in peer assessment activities (2) The attitude of peer assessment had a significant positive impact on the MOOC learners' behavioral engagement. The more positive the attitude was, the more they were willing to spend time and energy on learning. In the end, the study put forward suggestions for online peer assessment activities from three aspects: potential stimulation, training, and timely regulation.
\end{abstract}

Keywords: behavioral engagement, peer assessment, online learning, MOOC, attitude

\section{互评态度对 MOOC 学习者行为投入影响的个案研究}

况姗芸 $1, a$, 徐志君 $1, b^{*}$

\author{
1 教育技术学, 华南师范大学, 广州, 中国 \\ a261869310@qq.com \\ b*469249541@qq.com
}

\section{摘要}

学生的在线行为投入是影响其学习绩效的重要因素, 也是实现深度学习的先决条件。已有研究证实同伴互评活 动提供了让学生参与形成性评价过程的机会, 增强学习者间的互动并促进有效学习, 然而在线学习更多依赖学 习者本身, 学习者的互评态度可能对互评过程及学习效果产生较大影响。本研究依托《移动学习理论、技术与 实践》MOOC, 采用问卷调查与访谈法, 探究互评态度对于 MOOC 学习者在线行为投入的影响。调查结果发 现: (1) MOOC 学习者对于同伴互评较为认可, 持积极肯定态度, 愿意主动参与同伴互评活动 (2) 同伴互 评的态度对 MOOC 学习者行为投入有显著正向影响, 互评态度越积极, 越愿意投入精力和时间进行学习。研 究最后从潜能激发、互评培训、及时调控三方面为在线互评活动提出建议。

关键词: 行为投入, 同伴互评, 在线学习, MOOC, 态度 


\section{1. 问题提出与相关研究综述}

大规模的在线公开课程 (MOOC) 作为扩展高 等教育机会的一种有效方式，能提供开放而灵活的 体验, 正在国内和国际教育中广泛传播 (Jung, 2018)。 2017 年, 全球为 8100 万学习者提供了 9400 多个 MOOC (Barba, 2020), 然而如此高的参与率背后却 是课程的高辍学率、低投入率 (Jordan, 2015)。学习 投入作为衡量学习质量的重要指标, 能够预测学习 者学习绩效, 为有效的教学干预提供参考 (Dotterer \& Lowe, 2011; Lee, 2014; Henrie et al., 2015)。其中, 行为投入指学生在线学习过程中的特定行为表现, 能够进行观察与量化, 关注行为表现是进一步探索 认知和情感体验的重要前提。

相关研究表明, 成员间有效交互行为对学习投 入、学习效果有显著正向影响, 能够提高课程完成 率和学习效果。同伴互评提供了让学生参与形成性 评价过程的机会, 增强学习者间的互动并促进有效 学习, 已经成为 $\mathrm{MOOC}$ 平台普遍采用的评价方式 (Goh, 2019)。由于时空准分离的特性, MOOC 教 师难以像传统课堂中实时指导和监控学习者, 成功 的 MOOC 学习更多地依赖学习者个人特质, 如态 度、自我效能感、动机等 (Barba, 2020)。从心理学 上看, 态度会影响个体的感知方式, 对学习行为产 生强大的影响 (Bernard et al., 2004; Arbaugh, 2010), 一定程度预示了同伴关系的变化 (Sprer, 2020), 学 生的态度可能会对学习行为产生强大的影响。现有 的同伴互评研究很少关注 MOOC 环境下学生的态 度、动机等心理特征。因此, 迫切需要相关研究来 帮助教师了解学生在 MOOC 中实施在线互评活动 时的态度及其对行为投入的影响, 这有利于进行更 加科学准确的学习预测和教学干预, 为改进在线评 价活动提供参考依据。

\section{2. 研究设计}

\section{1. 研究对象选取}

本研究以《移动学习理论、技术与实践》MOOC 第一期为载体, 学员均参与了同伴互评活动, 在学 员参与的课程群发放电子问卷, 共回收有效问卷 47 份。

\section{2. 研究思路和方法}

本研究的主要目标是探究 MOOC 学习环境下 同伴互评的态度对于其行为投入参与、坚持、专注、 交互四方面的影响, 以探索在线同伴互评活动的改 进策略, 提升学习绩效。

采用的研究方法是问卷调查法和访谈法, 数据 分析工具主要是 SPSS25.0, 利用问卷收集 MOOC 学 员同伴互评态度以及行为投入情况, 利用 SPSS 进 行相关分析、回归分析等探究两者关系。根据聚类
结果挑选不同行为投入水平的同学进行半开放性访 谈, 了解学习者互评态度及其行为的产生原因。

\section{3. 研究工具}

\section{3. 1. 在线同伴互评态度调查量表}

在线同伴互评活动的实施效果会受到学习者态 度的影响, 大部分学习者对同伴互评这种评价方式 持积极肯定的态度, 由于互评设计不科学、学生质 疑公正性等原因, 部分学习者对同伴互评的体验感 较差, 不乐意投入互评活动中 (Siow, 2015)。对学 习者评价态度进行评测有利于优化互评活动设计, 目前学者们大多采用问卷调查对学习者态度进行评 测 (Wen \& Tsai, 2006; Black, 2020)。

基于此，本量表参考是同伴互评中较为经典的 《在线同伴评价态度问卷》 (Wen \& Tsai, 2006), 共 包括 6 个题项, 前 4 题直接采用 Wen \& Tsai (2006) 的量表。由于面对不同类型的互评活动，学习者的 态度也有所差异 (张小艳, 2016), 因此结合 MOOC 两种互评类型补充了后 2 题, 关注学习者对不同互 评活动的态度差异。采用李克特 5 级量表计分, 从 1 到 5 分别表示“完全不同意”到“完全同意”，得分 越高, 表示在线学习者互评的态度越积极。经检验, 本量表 Cronbach's $\alpha$ 系数为 $0.915(>0.9), \mathrm{KMO}$ 值 为 $0.869(>0.8)$, Bartlett 检验显著, 表示具有良好 信效度。

\section{3. 2. 在线行为投入评价量表}

当前主流的在线行为投入调查方法主要分为两 类, 一是获取平台上学习者外显行为数据, 较为客 观, 但由于 MOOC 大规模、开放的特性, 在功能上 有较多限制, 无法准确获取个人完整的学习行为数 据。二是利用问卷调查方法, 能够较为快速地对调 查对象整体投入情况进行量化, 当前学者们构建了 众多经典评测量表, 为行为投入的量化研究奠定了 坚实的基础。李爽等(2015)基于 Fredricks 的研究, 结合远程学习特点构建了《远程学生行为投入分量 表》，被验证具有良好信效度。而后众多学者在李爽 的基础上进行修订，但也都涉及到参与、坚持、交 互和专注四维度,

本研究直接采用李爽的《远程学生行为投入分 量表》, 从参与 (3 个题项)、坚持 (2 个题项)、交 互 (3 个题项) 和专注 (1 个题项) 四个维度进行调 查, 共 9 个题项, 如表 2 所示。量表采用李克特 5 点计分法, 从 1 到 5 分别表示“完全不符合”到“完全 符合”, 得分越高, 表示在线学习者的行为投入度越 高。经检验, 本量表 Cronbach's $\alpha$ 系数为 $0.889(>0.8)$, $\mathrm{KMO}$ 值为 $0.851>0.8$, Bartlett 检验显著, 说明量表 具有良好信效度。 


\subsection{3. 访谈提纲}

基于聚类结果挑选高、中、低投入度学员各 2 名, 共计 6 名, 借鉴 Wen \& Tsai（2006）的访谈提 纲, 围绕学员的互评动机、互评偏好、对课程参与 行为的影响四方面展开半开放访谈。

\section{3. 个案实施结果分析}

\section{1. 皮尔逊积差相关分析}

以互评态度作为一面的变量, 以在线行为投入 的四维度（参与、坚持、交互、专注）分别作为另 一面变量, 利用 SPSS25.0 进行了相关分析, 结果如 表 1 所示:

表 1 两变量间的 Pearson 相关分析

\begin{tabular}{|c|c|c|c|c|}
\hline & 参与 & 坚持 & 交互 & 专注 \\
\hline 互评态度 & $0.754^{* *}$ & $0.713^{* *}$ & $0.681 * *$ & $0.529 * *$ \\
\hline
\end{tabular}

可见, 同伴互评态度与在线学习行为投入的参 与、坚持、交互、专注四维度均呈现显著的正相关 关系, 说明互评态度对在线学习者的在线行为投入 有较大影响, 可以作进一步的回归分析。

\section{2. 线性回归分析}

由散点图可知, 互评态度与 MOOC 学习者在线 行为投入可能存在线性关系, 适合做线性回归分析 (如图 1 所示)。

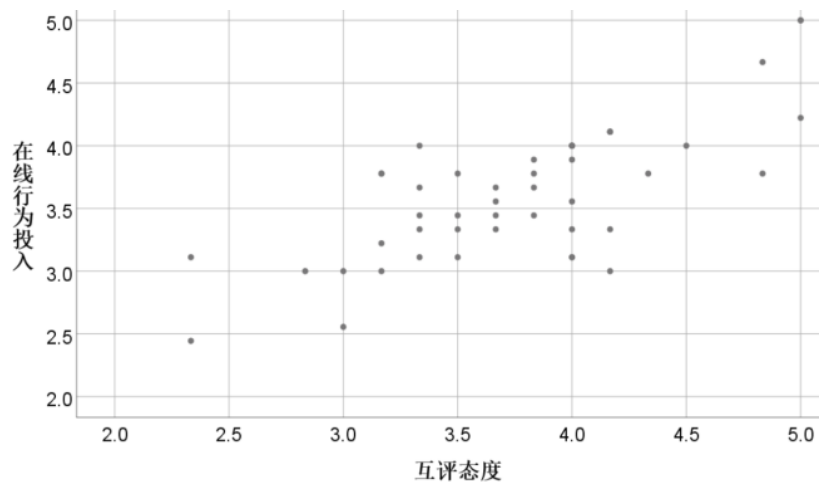

图 1 互评态度与在线行为投入关系散点图

将互评态度作为自变量, 而将在线行为投入度 作为因变量进行线性回归分析, 分析方法是选择“输 入”。所建立的回归模型 $1.8<\mathrm{DW}=2.111<2.2$, $\mathrm{R}^{2}=0.546$, 即学习者互评态度能解释其行为投入的 $54.6 \%$ 变化原因, 回归模型合理。从表 2 可知, 互评 态度的回归系数值为 $0.635(\mathrm{t}=7.359, \mathrm{p}=0.000<0.01)$, 意味着互评态度因素会对在线行为投入水平产生显 著的正向影响关系。读取未标准化系数, 本研究最 终所建立回归方程模型为 $\mathrm{y}=1.222+0.635 \mathrm{x}(\mathrm{x}=$ 互评 态度, $\mathrm{y}=$ 在线行为投入)。
表 2 线性回归分析结果

\begin{tabular}{|c|c|c|c|c|c|c|}
\hline \multicolumn{2}{|c|}{ 模型 } & \multicolumn{2}{|c|}{ 未标准化系数 } & $\begin{array}{c}\text { 标准化系 } \\
\text { 数 }\end{array}$ & $\mathrm{t}$ & 显著性 \\
\cline { 2 - 7 } & $\mathrm{B}$ & $\begin{array}{c}\text { 标准错 } \\
\text { 误 }\end{array}$ & Beta & & \multicolumn{2}{|}{} \\
\hline \multirow{2}{*}{1} & (常量) & 1.222 & .330 & & 3.705 & .001 \\
\cline { 2 - 7 } & 互评态度 & .635 & .086 & .739 & 7.359 & .000 \\
\hline
\end{tabular}

a. 因变量: 在线行为投入

\section{3. 访谈结果分析}

基于在线行为投入的 $\mathrm{K}$-means 聚类结果，挑选 高、中、低水平共 6 名学员围绕学员的互评动机、 互评偏好、对课程参与行为的影响展开深入访谈。

整理分析学生的访谈记录发现, (1) $83 \%$ 的同学 认为互评活动能够减弱孤独感, 督促他们参与课程。 1 位低投入的同学认为互评活动耗费了自己较多的 时间, 且同伴互评水平不等, 难以做到客观公正, 真正发挥同伴互评的作用。(2) 不同水平的学生也 表现出了对不同互评方式的偏好, 高投入的学生均 表示对论坛交流活动的偏好, 认为论坛间的相互交 流有助于促进反思, 而同伴评分活动不够准确反映 作品水平。低投入的学生偏好同伴评分活动, 认为 评分会与成绩挂钩, 促使他们花费更多时间去完成 作业以获得高分, 而在课程论坛与成绩关系不大, 因而会降低投入时间。(3) 所有学生均认为互评活 动能增加他们的学习参与。相比于教师批改, 在设 置有同伴互评批改要求的作业环节, 同学们在完成、 浏览、评价、完善作品上会花费较多的时间和精力, 行为投入度更高。在课程论坛中, 同伴互相评价、 点赞也促进了交互行为的发生，学生会投入更多时 间去浏览他人观点并进行反思。

综上, 参与调查的 MOOC 学员对同伴互评活动 持肯定态度, 愿意积极主动地参与互评, 并认为其 能促进自己的学习投入, 不同水平、动机的学员对 互评活动的喜好不一。

\section{4. 研究结论}

通过案例研究发现, 参与调研的 MOOC 学习者 普遍对同伴互评持积极肯定的态度, 认可同伴互评 的作用, 愿意主动参与同伴互评活动。MOOC 环境 中学习者的互评态度与其行为投入度显著正相关, 互评态度能解释其行为投入的 $54.6 \%$ 变化原因。研 究所建立回归方程模型为 $\mathrm{y}=1.222+0.635 \mathrm{x}(\mathrm{x}=$ 互评 态度, $\mathrm{y}=$ 在线行为投入), 学习者的互评态度越积极, 参与在线学习的频率越高, 行为投入度越高。这与 Prior (2016) 的观点 “学生的态度会影响自我效能, 进而影响到同伴参与行为” 和 Sedláek (2020) 提出 的观点“学生在课堂上的喜欢度和影响力越大, 他们 的参与度越高”相一致。而访谈则揭露了学生的互评 
动机及产生影响的原因, 同伴互评活动促使学习者 参与到形成性评价过程中，学生需要与同伴进行论 坛交流、批改作品等活动,而不仅仅停留在看视频、 文档等操作上，而观看同伴作品、收获同伴建议、 给予同伴建议能够促使学生自身进行反思与调控, 这就促进了学生的投入。

此外, 从 MOOC 论坛中学生的讨论行为数据也 显示, 在有同伴互评活动要求的前三章, 学生之间 的参与、交互行为频繁, 而在没有互评要求的后两 章节, 学生的参与度明显下降, 论坛中略显冷清, 这也侧面说明同伴互评能够促进学生学习参与。

根据分析结果, 对改善在线互评活动设计, 提 高 MOOC 学习者行为投入提出几点建议: (1) 根据 维果斯基的“最近发展区理论”, 学生现有的发展水 平和可能发展水平 (即潜力) 之间的差异就是最近 发展区。互评活动中要注着眼于学生的最近发展区, 活动前、中、后期应该对学生的态度及能力水平进 行阶段调查, 以此为依据确定下一阶段评价活动的 难度, 有效调动学生的积极性, 发挥其潜能。对于 表现突出的学员, 应该及时进行表扬, 发挥榜样示 范作用。(2) MOOC 学员背景迥异, 知识差距大, 在开展同伴评分活动前应该做好互评培训, 制定好 评价量规并进行试评活动。(3) 教师要对课程互评 情况有宏观把握, 可以利用 MOOC 平台记录的行为 数据 (如论坛发帖情况、同伴评分参与人数、视频 资料观看情况等) 进行监控, 比如若发现论坛中学 员讨论交互的频率下降, 应该及时采取激励措施, 调整教学计划。

由于研究精力有限, 本研究为个案研究, 样本 量较小且尚未能结合认知投入、情感投入全方面对 学习投入进行测量, 后续研究将扩大研究样本量, 增加认知和情感方面的指标进行综合评价, 进一步 挖掘互评态度与学习者 MOOC 学习投入各要素的 关系, 设置合理有效的同伴互评活动, 以提高学习 者 $\mathrm{MOOC}$ 学习参与度, 降低辍学率。

\section{项目基金}

本文为广东省教育教学改革项目《融入 “同伴 互评”的在线开放课程混合式教学改革与实践创新》 的阶段性成果之一。

\section{REFERENCES}

[1] Jung, Y., Lee, J. (2018) Learning engagement and persistence in massive open online courses (moocs). Computers \& Education, 122: 9-22.

[2] Barba, P. D., Malekian, D., Oliveira, E. A., Bailey, J., Ryan, T., Kennedy, G. (2020) The importance and meaning of session behaviour in a mooc. Computers \& education, 146: 103772.1103772.18 .
[3] Jordan, K. (2015) Massive open online course completion rates revisited: assessment, length and attrition. International Review of Research in Open and Distance Learning, 16: 341-358.

[4] Dotterer, A. M., Lowe, K. (2011) Classroom context, school engagement, and academic achievement in early adolescence. Journal of Youth and Adolescence, 40: 1649-1660.

[5] Lee, J.-S. (2014) The relationship between student engagement and academic performance: Is it a myth or reality? The Journal of Educational Research, 107:177-185.

[6] Henrie, C. R., Halverson, L. R., Graham, C. R. (2015) Measuring student engagement in technology-mediated learning: A review. Computers \& Education, 90: 36-53.

[7] Goh, C. F., Tan, O. K., Rasli, A., Choi, S. L. (2019) Engagement in peer review, learner-content interaction and learning outcomes. The International Journal of Information and Learning Technology. 36: 423-433.

[8] Bernard, R. M., Abrami, P. C., Y Lou, Borokhovski, E., Huang, B. (2004) How does distance education compare with classroom instruction? a meta-analysis of the empirical literature. Review of Educational Research, 74: 379-439

[9] Arbaugh, J. B. (2010) Sage, guide, both, or even more? an examination of instructor activity in online mba courses. Computers \& Education, 55: 1234-1244.

[10] Sprer, N., Lenkeit, J., Bosse, S., Hartmann, A., Knigge, M. (2020) Students' perspective on inclusion: relations of attitudes towards inclusive education and self-perceptions of peer relations. International Journal of Educational Research, 103: 101641.

[11] Siow, L. F. (2015) Students' perceptions on selfand group-assessment in enhancing learning experience. Malaysian Online Journal of Educational Sciences, 3: 21-35.

[12] Wen, M.-L., Tsai, C.-C. (2006) University students' perceptions of andattitudes toward (online) peer assessment. Higher Education, 1: 2744.

[13] Black, E. W., Dickson, T., Blue, A. V. (2020) Exploring item discrimination in an online self and peer assessment of interprofessional teamwork. Journal of Interprofessional Education and Practice, 22: 100396. 
[14] Li, S., Yu, C. (2015) Development and Implementation of Distance Student Engagement Scale. Open Education Research, 21: 62-70+103.

[15] Prior, D. D., Mazanov, J., D Meacheam, Heaslip, G., Hanson, J. (2016) Attitude, digital literacy and self efficacy: flow-on effects for online learning behavior. Internet \& Higher Education, 29: 91-97.

[16] Sedláek, M., Eova, K. (2020) Are student engagement and peer relationships connected to student participation in classroom talk?. Learning Culture and Social Interaction, 26: 100411. 